\title{
Das Verhältnis von Macht, Geschlecht und (Dis-)Ability in der Forschung
}

\section{Dagmar Kubanski \& Stephanie Goeke}

\author{
Journal für Psychologie, 26(2), 95-113 \\ https://doi.org/10.30820/8248.06 \\ www.journal-fuer-psychologie.de
}

\section{Zusammenfassung}

Partizipative Forschung wird im Kontext der Teilhabediskussion nach Ansicht der Autorinnen nicht konsequent diskutiert. Rechte nach dem Bundesteilhabegesetz kaschieren unsichtbare Machtdynamiken. Im Aktionsbündnis Teilhabeforschung engagieren sich Personen sowie Organisationen aus unterschiedlichsten gesellschaftlichen Bereichen und Forschungsgebieten, um das Ziel zu verfolgen, ein neues Forschungsfeld zu entfalten. Der Beitrag greift die Machtfrage auf, einerseits in ihrer Gewaltförmigkeit und andererseits als positiv konnotierte Gestaltungsmacht, die insbesondere gesellschaftlich benachteiligten Bürger_innen abgesprochen wird. Indem Ableism als Erklärungskonzept und explizit Frauen, Männer und Transmenschen mit Behinderung in den Blick genommen werden, wird die Konstruktion von Wirklichkeit und Wahrheit und deren Bedeutungszuschreibungen betrachtet. Dabei werden Macht, symbolische Gewalt und Geschlecht als bedeutsame Kategorien für die Analyse herausgestellt, um unsichtbare Strategien, Techniken und Versprechen sichtbar werden zu lassen. Eine Skizzierung der unterschiedlichen partizipativen Forschungszugänge soll einen Einstieg in die Auseinandersetzung des Einbezugs von Menschen mit Behinderung in Forschungsprozesse bieten. Zudem wird anhand exemplarischer Beispiele verdeutlicht, dass partizipative Forschung auf emanzipatorischen und empowernden Prozessen basiert und einer intersektionalen Betrachtungsweise bedarf.

Schlüsselwörter: Partizipative Forschung, Macht, Ableism, Intersektionalität, Geschlecht, Selbstermächtigung

\section{Summary}

The relationship of power, gender and (dis-)ability in the research

Participatory research is not consistently discussed in the context of the debate of participation in the authors' opinion. Rights under the »Bundesteilhabegesetz « [The Social Security Code IX: Rehabiliation and Participation of Disabled People] conceal invisible power dynamics that may show up in a lack of recognition or appreciation. In the $\gg$ Aktionsbündnis Teilhabeforschung « [Alliance of People: Participation research] individuals as well as or- 
ganizations from different social sectors and research areas have committed themselves to pursue the goal of developing a new field of research. The contribution addresses the question of power, on the one hand in its acts of violence and on the other as positively connotated creative power, which is particularly denied to socially disadvantaged people. Looking at the conception of ableism and especially to women, men and transpersons with disabilities, the construction of reality and truth and their attributions of meaning are considered. Power, symbolic violence and gender are important categories for analysis to make invisible strategies, techniques and promises visible. A delineation of the different participatory research approaches should provide an introduction to the debate of an involvement of people with disabilities in research processes. It is illustrated by exemplary examples that participatory research is based on emancipatory and empowering processes and an intersectional approach is needed.

Keywords: participatory research, power, ableism, intersectionality, gender, empowerment

\section{Einführende Gedanken}

In unserer Veröffentlichung »GrenzgängerInnen im akademischen Raum «(Goeke \& Kubanski, 2012) konnten wir bereits zeigen, welche Formen des Einbezugs von Menschen mit Behinderung sich in Forschungsprozessen finden. Damit bot der damalige Beitrag die Chance, vor dem Hintergrund einer gründlichen Recherche vorhandener Ansätze den Kreis forschender Menschen zu erweitern und damit den akademischen Raum für das Thema zu öffnen. Auch wenn der Einbezug von Expert_innen in eigener Sache selbstverständlicher wird, gibt es Leerstellen, die es kritisch zu beleuchten gilt. In diesem Beitrag möchten wir zeigen, dass im Kontext der Betrachtung von Ungleichheit nicht nur (Dis-)Ability bedeutsam ist, sondern auch das Geschlecht derzeit nicht ausreichend im Diskurs Berücksichtigung findet. Außerdem werden Machtverhältnisse oftmals negiert und kommerzielle Interessen im Kontext der Leistungserbringung zu wenig gesehen (Gerhards, 2013). Der vorliegende Artikel will somit einerseits auf die Verwobenheit von Gender und Behinderung, andererseits auf die - aus unserer Sicht bisher wenig reflektierte - Verflechtung von Macht, (Dis-)Ability und Geschlecht Bezug nehmen und dies in den Kontext partizipativer Forschung stellen.

Dieser Beitrag nimmt Frauen, Männer und Transmenschen mit Behinderung in den Blick und betrachtet die Konstruktion von Wirklichkeit und Wahrheit und deren Bedeutungszuschreibungen (Bargetz et al., 2017). Diese Zusammenhänge sind in Forschungsprozessen durchaus bekannt, müssen aber immer wieder neu in den Vordergrund gestellt werden, da ihnen zentrale Bedeutung zukommt. 
Vorausgeschickt wird, dass die Begriffe Behinderung, Beeinträchtigung, (Dis-)Ability und Betroffene von uns aufgegriffen und bei Bedarf kritisch hinterfragt werden. Leser_innen mögen sich auf den Weg begeben, Sprache nicht nur als Mittel zu betrachten, um Realität zu beschreiben, sondern als etwas zu sehen, womit Realität geschaffen wird.

In unterschiedlichen Zusammenhängen werden Frauen, Männer und Transmenschen mit Behinderung unterschiedlich benannt und dabei meist auf ihr Behindertsein als einziges Heterogenitätsmerkmal reduziert. Dagegen findet sich kaum eine konsequentere Betrachtung aus intersektionaler Perspektive, die auf der Darstellung von Machtkonstruktionen basiert und die Verwobenheit und Wechselwirkung sozialer Kategorien wie Geschlecht, Klasse, »Rasse«, Ethnizität usw. analysiert. Hierbei wird wiederum allzu oft die Kategorie Behinderung ausgeblendet (Raab, 2010); zudem wird der Begriff Gender vorrangig mit Frauen assoziiert.

In der Umsetzung einer geschlechtergerechten und diversityorientierten Sprache verwenden wir den Gender_Gap und drücken damit aus, dass es jenseits der Zweigeschlechtlichkeit noch weitere Konstruktionen von Geschlecht geben kann, und schließen Menschen ein, die sich dem Geschlecht Mann oder Frau nicht zuordnen möchten.

Der Beitrag beginnt mit einer Beschreibung des partizipativen Forschungsfeldes und gibt einen Überblick über verschiedene Forschungszugänge. Im Anschluss wird das Erklärungskonzept Ableism eingeführt, um Erwartungen, die an Menschen gestellt werden, gesellschaftlich einzuordnen. Dieses Konzept bietet Anschlussmöglichkeiten an das kulturelle Modell von Behinderung und könnte ein Instrument zur Reflexion partizipativer Forschung sein. Hochschulen sind für uns ein gesellschaftspolitisches normengeprägtes Feld, in welchem Machtasymmetrien täglich und institutionell legitimiert gelebt werden. Ausgehend von einem Mangel an symbolischem Kapital (Goeke, 2010; Kubanski, 2013), welches Menschen mit Behinderung zur Verfügung steht, und davon, dass Akte der Exklusion der symbolischen Gewalt zugeordnet werden können, wird - Pierre Bourdieu (1998, S. 173f.) folgend - dieser Zusammenhang als Erklärung für Machtverhältnisse weiter ausgeführt. Abschließend werden mögliche Ausformungen partizipativer Forschung und die dafür erforderlichen Rahmenbedingungen in den Blick genommen.

Im dem nachfolgenden Teil wird ein kurzer Blick hinter die Kulissen unterschiedlicher Forschungszugänge und deren Rahmenbedingungen geworfen. Dies geschieht in erster Linie mit dem Mittel der Literaturrecherche, aber auch vor dem Hintergrund eigener Erfahrungen und Aktivitäten in Netzwerken und Hochschulen. Das Ziel ist, kaum sichtbare Praxen von Machtasymmetrien zu skizzieren, denn die Bereitschaft zu deren Aufdeckung bildet die Basis für ein Verständnis partizipativer Forschung. 


\section{Das partizipative Forschungsfeld - eine aktuelle Situationsbeschreibung}

Partizipative Forschung wird im Kontext der modernen Teilhabediskussion nicht konsequent genug diskutiert. Denn Ziele, wie z.B. die Förderung der Teilhabe am Arbeitsleben oder die Verbesserung der Lebenssituation, deren Umsetzung das Bundesteilhabegesetz ermöglichen soll, kaschieren Machtdynamiken, die sich möglicherweise in fehlendem gesellschaftlichen Status zeigen. Symbolisches Kapital, wie etwa Wertschätzung und Anerkennung, stellt eine Art »Kredit « dar, den die Empfänger_innen bedingungslos erhalten (Bourdieu, 1993, S. 218). In Verbindung mit einer zugeschriebenen Behinderung erhalten diese Frauen und Männer meist symbolisches Kapitel nur durch besondere Anstrengungen.

Aus unserer Perspektive könnte hier partizipative Forschung, die auf einem emanzipatorischen Prozess basiert, zu einem Mehr an Inklusion und Gestaltungsmacht verhelfen. Die Vielfalt im Hinblick auf das Thema Inklusion und Teilhabe, die sich derzeit in hochschulischen Fachbereichen, Arbeitsschwerpunkten oder Netzwerken zeigt, ist möglicherweise ein Schritt auf diesem Weg. Es scheint aber besondere Aufmerksamkeit notwendig, damit unterschiedliche Interessen aufgedeckt werden. Hier könnten Fremdinteressen, wie z. B. bei der durch Firmen mitfinanzierten Versorgungsforschung, kommerzieller Natur sein (Mehrholz, 2018). Indem Leistungserbringer im Bereich der Rehabilitation Mobilität und damit unter anderem auch Teilhabe definieren, kann Selbstbestimmung, etwa bei der Bewilligung von Hilfsmitteln oder Assistenz, in eine bestimmte Richtung gelenkt werden. Zudem bestehen Interessenslagen bei Forscher_innen in Bezug auf die Frage, wie mit ihren Wissensproduktionen verfahren werden sollte.

In den letzten Jahren hat die Zahl der Lehrstühle im Kontext Inklusion zugenommen. Dies bestätigt eine systematische Internetrecherche für den Bereich der deutschen Hochschulen mit den Suchbegriffen »Inklusion, Professur, Lehrstuhl «. Dabei zeigt sich, dass die zahlreich in den letzten Jahren berufenen Inklusionsprofessor_innen sowie die ausgeschriebenen Lehrstühle eine große Heterogenität im Profil aufweisen. Die Spannbreite reicht von Bildung über Organisationsentwicklung bis hin zu Pädagogik oder spezifischen Förderschwerpunkten. Jedoch ist dabei eine Reduktion auf die Kategorie Behinderung erkennbar. Dies wird deutlich z. B. durch klassische sonderpädagogische Fragestellungen der Forschungsprojekte oder an sehr spezifischen Stellenprofilen der Professuren. So zugeschnittene Stellen verunmöglichen unseres Erachtens emanzipatorische Aktivitäten von Hochschulangehörigen (mit Behinderung). Wir vermissen eine intersektionale Forschungsperspektive, die aber notwendig wäre, um mögliche Forschungsgegenstände umfassender zu ergründen.

Auf einer anderen Ebene liegt die Frage nach dem Zustandekommen partizipativer Forschungszusammenhänge: Können ermächtigende Praxen in androzentrischen 
Institutionen unter dem Einfluss von Sparpolitik realisiert werden? Forschung steht an Hochschulen in ihrer Ausrichtung oftmals unter dem Kalkül von Effizienz und Exzellenz, sodass betriebswirtschaftliche Überlegungen die Entscheidung der Auswahl von Forschungsprojekten mit steuern. Diese Situation erschwert es, neue Räume für ein partizipatives Forschungsfeld zu eröffnen sowie neue Wege des Denkens zu gehen (Ludwig, 2011, S. 118f.).

Es besteht außerdem noch in Fachkreisen die Befürchtung, dass spezifische Ressourcen wie heilpädagogische Förderung, fundierte Diagnostik oder sonderpädagogische Begleitung bei der Umsetzung von Inklusion zu kurz kommen könnten (Reichenbach, 2009). Zudem zeigen sich berufsständische Interessen, die den Entwicklungsprozess möglicherweise beeinflussen (Hinz \& Köpfer, 2016).

Immer noch haben es die Disability Studies, trotz eigener Lehrstühle, schwer, ihren Stand in der deutschsprachigen scientific community einer größeren Öffentlichkeit zu vermitteln. Marianne Hirschberg (2015) spricht zwar davon, dass bereits durch die Disability Studies ein Paradigmenwechsel »von der Fürsorge zur Teilhabe in der Behindertenpolitik « stattfand. Diese Einschätzung ist optimistisch und spiegelt unseres Erachtens nicht die Gesamtlage in der deutschen Hochschullandschaft wider. Neben wenigen Lehrstühlen wird die Arbeitsgemeinschaft Disability Studies in Deutschland weitestgehend ehrenamtlich getragen, was aufgrund der begrenzten Ressourcen öffentliche Präsenz und Handlungsmöglichkeiten einschränkt (vgl. Ev. Hochschule RheinlandWestfalen-Lippe Informationsplattform BODY, 2015; Arbeitsgemeinschaft Disability Studies Deutschland, 2018). Im Folgenden werden zentrale methodologische Zugänge der partizipativen Forschung beschrieben, denn auf dieser Basis ist es möglich, ein Verständnis für die Aktualität machtvoller akademischer Wissensproduktion zu erzeugen, die häufig weit weg von der Lebensrealität behinderter Menschen stattfindet (Buchner \& Koenig, 2013, S. 177).

\section{Partizipative Forschungszugänge}

Aktuell finden sich im deutschsprachigen Raum unterschiedliche Begrifflichkeiten in Bezug auf die Teilhabe von Menschen mit Behinderung an Forschungsprozessen, die sich teils an internationale Bezeichnungen anlehnen. Eine kurze Erläuterung der Zugänge ist wichtig, da dabei die Radikalität sowie die Konsequenzen der unterschiedlichen Vorgehensweisen deutlich werden. Denn bei partizipativer Forschung handelt es sich um ein weitverzweigtes Feld mit den verschiedensten methodischen Zugängen (von Unger, 2014).

Im internationalen Diskurs gibt es unter anderem die Forschungszugänge Emancipatory Research, Participatory Action Research (PAR) und Inclusive Research. Diesen 
Forschungszugängen kommt zunehmend mehr Aufmerksamkeit im öffentlichen und wissenschaftlichen Diskurs zu, von einem Durchbruch kann jedoch nicht die Rede sein.

Emancipatory Research stellt die radikalste Form partizipativer Forschung dar, und ist nach Gerry Zarb (1992) klar abzugrenzen von Participatory Research, weil Kontrolle, Macht und Entscheidungsgewalt in den Händen von Frauen und Männern mit Behinderung liegen und diese und ihre Organisationen die Kontrolle über die Forschung und ihre Ziele haben. Wissenschaftler_innen ohne Behinderungen übernehmen die Rolle der Berater_innen. $\gg$ The two primary principles of $>$ empowerment< and $>$ reciprocity<, which are the heart of present debates about emancipatory research « (ebd., S. 127), müssen nach Zarb so verstanden werden - und hier schließt er sich Michael Oliver an -, dass zentral ist, was Forschung für die Unterstützung des Empowermentprozesses tun kann. Partizipatorische Forschung ist somit ein Schritt bzw. eine Voraussetzung auf dem Weg zu emanzipatorischer Forschung.

Der im Kontext der US-amerikanischen Independent Living-Bewegung diskutierte Begriff Participatory Action Research (PAR) wird im Deutschen als partizipative Handlungsforschung bezeichnet. PAR basiert auf wesentlichen Merkmalen der Aktionsforschung, z. B. der Intention, Praxis zielbezogen zu verändern. PAR versteht sich als eine grundsätzliche Haltung in Bezug auf Machtverhältnisse und die Reproduktion von Kategorien, die eigene Rolle im Forschungshandeln zu reflektieren. Dabei legt PAR »einen stärkeren Akzent auf die Beteiligung von Teilnehmer/innen eines Feldes oder einer Organisation am Erkenntnisprozess, und die beteiligten Wissenschaftler/innen haben Beratungsfunktion « (von Unger, 2014, S. 21).

Der Terminus Inclusive Research (Inklusive Forschung) wurde von Jan Walmsley (2001) als Reaktion auf die strittige Frage der Besitzrechte an und der Kontrolle von Forschung geprägt. Inklusive Forschung wird dabei verstanden als ein Set von Kategorien, mit dem sowohl die partizipatorische als auch die emanzipatorische Forschung im Kontext von Lernschwierigkeiten eingeschlossen wird (Walmsley \& Johnson, 2003). Tobias Buchner, Oliver Koenig und Saskia Schuppener (2016) verdeutlichen, dass es um die Partizipation von Menschen mit Lernschwierigkeiten geht. Die Herausgeber_innen des Buches Inklusive Forschung. Gemeinsam forschen mit Menschen mit Lernschwierigkeiten sind sich dieser Engführung auf den Personenkreis der Frauen und Männer mit Lernschwierigkeiten bewusst, erachten diese Fokussierung aber zugleich als unabdingbar, um einen Diskurs im deutschsprachigen Raum zu führen (ebd., S. 14).

Inklusionsforschung, Netzwerke und Tagungen machen deutlich, dass der Fokus im deutschsprachigen Raum auf Kinder und Jugendliche, Lehrkräfte und Erzieher_innen im schulischen bzw. pädagogischen Kontexten gelegt wird. Seit über 30 Jahren kommen Integrations-/Inklusionsforscher_innen zu ihrer Jahrestagung zusammen. In den Tagungsbänden der letzten 15 Jahre dominieren allerdings, neben heilpädagogischen Fra- 
gestellungen, überwiegend Forschungsfragen des schulischen Kontextes die Diskurse. Zugleich gründen sich bundesweit Netzwerke, Bündnisse und Arbeitsgemeinschaften unter dem Label Inklusionsforschung. So hat unter anderen der Vorstand der Deutschen Gesellschaft für Erziehungswissenschaften (DGfE) in seiner Sitzung am 3./4. November 2016 eine Arbeitsgruppe Inklusionsforschung für drei Jahre eingerichtet.

User-Led-Research (Betroffenen-kontrollierte Forschung) ist ebenfalls eine gängige Bezeichnung, um zu verdeutlichen, dass die Forschungsverantwortung von betroffenen Menschen selbst übernommen wird. Jasna Russo (2012) sieht dies z. B. als weitestgehende Entwicklung des partizipativen Ansatzes im psychiatrischen Bereich.

Die vor allem in den Sozial- und Kulturwissenschaften verankerten Disability Studies verbinden mit Forschung unter anderem das Ziel, ausgrenzende Praxis aufzuzeigen, $\mathrm{zu}$ analysieren und zu beseitigen. In ihrem Verständnis haben viele Akteure und Akteurinnen, die Wissenschaftler_innen selbst, eine Behinderungserfahrung und vertreten einen emanzipatorischen Forschungsansatz (Zander, 2016). Dabei werden die Themen Inklusion und Teilhabe sowie gesellschaftliche Ausgrenzung zum Forschungsgegenstand und die Disability Studies setzen auf Partizipation am und Empowerment der Menschen mit Behinderung im Forschungsprozess. Mit dem sozialen und kulturellen Modell von Behinderung grenzen sich die Disability Studies explizit vom medizinischen Modell von Behinderung ab.

Transdisziplinäre Forschung ist im deutschsprachigen Raum in einigen Wissenschaftsbereichen als Terminus zu finden (Ministerium für Kultur und Wissenschaft des Landes Nordrhein-Westfalen, 2018). Ausgehend von gesellschaftlichen Problemstellungen versucht die transdisziplinäre Forschung wissenschaftliches und praktisches bzw. nicht-akademisches Wissen zu verknüpfen, indem verschiedene Fachdisziplinen gemeinsam forschen, um schließlich zu einer am Gemeinwohl orientierten Lösung zu gelangen. Im Kontext transdisziplinärer Forschung wurden bereits Netzwerke gegründet, um die Entwicklung von Qualitätskriterien voranzutreiben. Das Bildungs- und Forschungsministerium nutzt diesen Terminus für partizipativ angelegte Forschungsprojekte des Bundes.

Teilhabeforschung ist eine noch relativ junge Richtung in Deutschland. Im Juni 2015 gründete sich das Deutsche Aktionsbündnis Teilhabeforschung, um Teilhabe auf der Grundlage der UN-Behindertenrechtskonvention (UN-BRK) zu fördern. In Artikel 4 Allgemeine Verpflichtungen finden sich die Bereiche Forschung sowie Entwicklung und Artikel 31 trifft Aussagen zu Statistik und Datensammlung. Teilhabeforschung wird als Querschnittsdisziplin verstanden, soll interdisziplinär, transdisziplinär, international, intersektional orientiert sein und die Heterogenität der Menschen mit Behinderungen berücksichtigen - so Anne Waldschmidt (2015). Sie hebt hervor: »Ganz wichtig ist auch, dass wir bei allem, was wir tun, partizipativ orientierte Forschungsmethoden durchführen und anwenden « (ebd., S. 687). 
Aktuell formieren sich im Kontext der Aktivitäten des Aktionsbündnisses Teilhabeforschung engagierte Personen in Arbeitsgruppen sowie Organisationen aus unterschiedlichen gesellschaftlichen Bereichen und Forschungsgebieten, um gemeinsam das Ziel zu verfolgen, neue Forschungsfelder zu entfalten. Die Forschungsaktivitäten zu Lebenslagen von Menschen mit Behinderung sollen zudem gestärkt, gebündelt und sichtbar gemacht werden. Dabei können Menschen mit Behinderungen Wege zu partizipativer Forschung eröffnet werden (siehe www.teilhabeforschung.bifos.org).

Partizipative Forschung wird sowohl von Jarg Bergold und Stefan Thomas (2012) als auch von Hella von Unger (2014) als Forschungsstil verstanden. Sie speist sich aus einer Vielzahl von Forschungsmethoden und Forschungsstrategien, die die Partizipation der Ko-Forscher_innen fördern und fordern will und stellt einen eigenständigen wissenschaftlichen Forschungsansatz dar. »Die Vielfalt ist Programm, wodurch sie sich einer vereinheitlichenden Methodologie entzieht « (von Unger, 2014, S. 101).

Die Gemeinsamkeit der hier skizzierten methodischen Zugänge liegt darin, dass tradierte Grenzen von Praxis und Wissenschaft aufgehoben werden. Alle Zugänge haben Partizipation im Forschungsprozess als Kernmerkmal, d. h. Teilhabe an Wissensproduktion und Theoriebildung. Entlang eines sozialen und kulturellen Modells von Behinderung und Geschlecht werden Frauen und Männer mit Behinderungserfahrung, Patient_innen und Betroffene als Subjekte wahrgenommen. Forschen wird als gemeinsamer Lernprozess verstanden und Forschung soll einen Beitrag zur Veränderung der Lebensverhältnisse der Betroffenen leisten sowie die Lebensqualität in den Mittelpunkt der Betrachtung stellen. Oftmals geht es weniger um verallgemeinerbare Ergebnisse als um den Erkenntnisweg während des Forschungsprozesses. Bei Tina Cook et al. (2018) heißt es:

»For most staff improving communication was not a contested aspiration. Understanding the nature of communicative endeavor for inclusive practice was, however, complex. A key issue for staff was not lacks of intent communicate, but that they were not sufficiently aware of how the complexities of power, hierarchy, positioning and ownership of the space could influence an encounter « (Paragraf 41).

In ihrer partizipativen Forschung zur inklusiven Praxis unterschiedlicher Unterstützungssysteme für langfristig neurologisch erkrankte Menschen gelingt es Cook et al. so, zentrale Einflussfaktoren für gelingende Zusammenarbeit zu identifizieren. Die Forschungszugänge unterscheiden sich zwar hinsichtlich ihrer Eindeutigkeit, Menschen mit Behinderung als Ko-Forscher_innen einzubeziehen, dennoch sind damit die Hoffnung auf Demokratisierung und Empowerment der Menschen mit Behinderung verbunden. Allerdings finden sich in der deutschsprachigen Literatur bisher wenig Auseinandersetzungen mit Fragen von Konflikten und Macht in partizipativen For- 
schungsprozessen. Auch deshalb macht von Unger (2014, S. 88ff.) auf die Bedeutung der (Selbst-)Reflexivität der Forschenden aufmerksam und fordert, ethische Prinzipien deutlicher zu berücksichtigen, die in Großbritannien für community-basierte partizipative Forschung erarbeitet wurden. Swantje Köbsell (2010, S. 25) thematisiert das Verhältnis von Geschlecht, Behinderung und Körper und macht auf die Diskurse in Großbritannien aufmerksam, in denen behinderte Frauen die Unzulänglichkeiten des sozialen Modells benannten und forderten, »die erlebten körperlichen Erfahrungen mit Beeinträchtigung und Behinderung in den Diskurs miteinzubeziehen « und somit » auch >Impairment< als sozial konstruierte Kategorie an[zu]sehen «. Sie merkt an, dass im Konzept der Intersektionalität die Kategorie Behinderung selten einbezogen ist (ebd.).

Für die Frage von Macht und Empowerment ist entscheidend, sich selbst als Lernende zu verstehen und die Expertise anderer gleichermaßen wertzuschätzen. Das impliziert ein neues Forschungsverständnis und das Aufgeben von Privilegien. Im Kontext der oben angeführten partizipativen Forschungszugänge dient Forschung nicht nur der Produktion von Wissen, sondern zielt auch auf die Veränderung der Umgangsformen untereinander ab. Außerdem wandeln sich im besten Fall die Lebensbedingungen der Betroffenen. Wo jedoch bestehen mögliche Grenzen der Einbeziehung von Laien, Betroffenen oder Menschen mit Unterstützungsbedarf in Forschungsprozesse?

So verdeutlicht z. B. die Debatte zwischen dem Physiologen und ehemaligen Präsidenten der Union der deutschen Akademie der Wissenschaften Günter Stock und dem Wirtschaftswissenschaftler Uwe Schneidewind (Grefe \& Sentker, 2014) den Streit um das Mitspracherecht in Forschungsprozessen sowie die Verwendung von Fördermitteln und zeigt die Sorge, dass gesellschaftliche Einzelinteressen die Grundlagenforschung schwächen könnten. Andererseits wird sichtbar, wie wichtig die Perspektiverweiterung ist und wie sehr derzeit die immer gleichen Netzwerke aus Wissenschaft, Politik und Industrie die Forschungsförderung beeinflussen, wie Schneidewind (2014, o. S.) darlegt. Die genauere Betrachtung der Argumentation zeigt, dass es Stock um die Grundlagenforschung geht und Schneidewind die angewandte Forschung im Blick hat. Für Schneidewind steht die folgende Frage im Fokus: »In welcher Form ließe sich Zivilgesellschaft optimal beteiligen? Wie organisieren wir wirkliches Querdenken? « (Grefe \& Sentker [im Interview mit Stock \& Schneidewind], 2014)

Fragen der Inklusion und Exklusion im Wissenschaftskontext berühren zusätzlich die Frage des Wissenschaftsverständnisses und des Selbstverständnisses der Wissenschaftler_innen. Wie ist partizipative Forschung im Wissenschaftskanon verortet? Dieser Frage geht von Unger (2014) nach und kommt zu dem Schluss, dass partizipative Forschung im deutschsprachigen Raum noch wenig Unterstützung erfährt. Hella von Unger zeigt zwar, welche Entwicklungen sich vollzogen haben, dennoch besteht weiterhin eine »verkürzte Wahrnehmung von partizipativer Forschung als einem ge- 
scheiterten Versuch aus den 1970er-Jahren [...]. Darüber hinaus bestehen Einwände gegenüber bestimmten methodologischen Grundsätzen der partizipativen Forschung « (ebd., S. 98). Dies erschwert eine breitere Akzeptanz partizipativer Forschungszugänge.

Möglicherweise müssten sich Wissenschaftler_innen selbstkritisch fragen, welche Bilder von Behinderung sie (re-)produzieren und wie diese in Diskurse der Normierung und Normalisierung verwoben sind. »Für die Disability Studies bedeutet dies, dass > Soziale Arbeit < im Spannungsfeld von Handlungsmächtigkeit, im Sinne partizipatorischer, inklusiver Ermöglichung und einer gouvernementalen Normierungs- und Normalisierungstechnologie angesiedelt ist (Mecheril \& Melter, 2012, 264) (zit.n. Raab, 2015, o. S.). Das Konzept des Ableism könnte für diese notwendige Reflexion ein geeigneter Ausgangspunkt sein.

\section{Fähigkeitserwartungen und das Konzept des Ableism}

Menschen werden in unserer Gesellschaft zumeist mit ihren körperlichen und kognitiven Fähigkeiten wahrgenommen. Es wird gesellschaftlich erwartet, dass sie leistungsfähig und produktiv sind und bestimmte Bildungsabschlüsse vorweisen können. Landläufig werden Menschen, die auf umfangreiche Unterstützungsleistungen angewiesen sind, nicht mit dem Leistungsprinzip in Verbindung gebracht - außer sie sind zu überdurchschnittlichen Denkleistungen fähig, wie der Physiker Stephen Hawking, oder zeigen im paralympischen Sport, wie sie mittels Höchstleistungen ibr Schicksal meistern. Die beiden plakativen Beispiele sollen helfen, sich dem Konzept Ableism zu nähern, das aufzeigt, wie dominierend Vorstellungen der Selbstoptimierung sein können. Mit dem Konzept Ableism erfolgt eine Perspektivveränderung in den Disability Studies, das heißt, es wird nicht nur die Diskriminierung behinderter Menschen betrachtet, sondern die Dualität Behinderung und Nichtbehinderung aufgehoben, denn Fähigkeitserwartungen werden an alle Menschen gestellt.

Hier ist das kulturelle Modell von Behinderung anschlussfähig, so wie es unter anderen Waldschmidt (2005) in die Disability Studies einbringt. Durch eine kulturwissenschaftliche Perspektive wird es möglich, Behinderung als Folge einer Zuschreibung und als Deutungsmuster innerhalb der Gesellschaft zu begreifen.

Entscheidend ist, dass Fähigkeiten den Bewertungsmaßstab bestimmen. »Nichtbehinderung und Gesundheit werden zum Ideal erhoben, an dem sich alle Körper zu bewähren haben « (Maskos, 2015, S. 2f.). Ableism ist somit »der treffende Begriff für die Reduzierung von Menschen auf ihren - nicht/behinderten - Körper. Ability bedeutet Fähigkeiten und Ableism die einseitige Fokussierung auf körperliche und geistige Fähigkeiten einer Person und ihre essentialisierende Be- und Verurteilung, je nach Ausprägung ihrer Fähigkeiten « (Maskos, 2010, S. 2). Ableism stellt für Rebecca Maskos ein 
Konzept dar, das bewusst Personen einschließt, die Eigenschaften zeigen, die als besonders positiv bewertet werden, z. B. wird blinden Menschen automatisch ein sehr gutes Gehör oder eine spezielle Fähigkeit des Fühlens zugesprochen. Lisa Pfahl (2014) hält fest: »Ob Menschen behindert oder befähigt werden, ist in erster Linie eine Entscheidung von Institutionen und den von ihnen vorgenommenen Grenzziehungen zwischen Personen, die den Menschen das Recht auf Freiheit, Gleichheit und Teilhabe - und ich würde ergänzen Produktivität - zugestehen, oder eben nicht.«

Für den Kontext Ableism und partizipative Forschung würde das bedeuten: Personen, denen per se Defizite oder bestimmte Rollen zugeschrieben werden, zu ermächtigen, sich in Forschungsprozesse einzubringen und diese gleichberechtigt als Expert_innen mitzugestalten. Für Forscher_innen heißt dies, aus ihrer privilegierten Position heraus zu treten und damit habituell begrenzte Räume zu verlassen. Im Wissenschaftsfeld könnte nach Bourdieu die strukturierende Struktur des Habitus ${ }^{1}$ wirksam werden, indem politische Instrumente als aufständische Positionen Veränderungen ermöglichen (Bourdieu, 1993, S. 113). Macht abzugeben heißt dann, Empowermentprozesse zu befürworten und zu befördern. Für die Forschung, meist regelhaft verortet an einer Hochschule oder zumindest eingebettet in einen institutionellen (akademischen) Kontext (Stiftungen, Forschungsinstitute), könnte die Anwendung des Konzepts Ableism unter anderem solche Veränderungen befördern.

\section{Formen von Machtstrukturen/-konstellationen im Forschungskontext}

Vor dem Hintergrund unserer bisherigen Ausführungen stellt sich die Frage, wie sich partizipative Forschung gestalten lässt, um sich selbstreflexiv Machtstrukturen zu nähern. Ausgehend davon, dass Akte der Exklusion behinderter Menschen nach Bourdieu (1998) der symbolischen Gewalt zugeordnet werden können, ist es im Kontext von Forschungszugängen und deren Ermöglichung wichtig zu verdeutlichen, dass Bourdieu das symbolische Kapital in Verbindung zur symbolischen Gewalt setzt. Er bezeichnet das symbolische Kapital als »eine beliebige Sorte von Kapital « (ebd., S. 150), da das ökonomische, soziale und kulturelle Kapital in einer bestimmten Konstellation zu symbolischem Kapital werden kann. Wenn also symbolisches Kapital mit Anerkennung umschrieben werden kann, so wirft die »Zugehörigkeit zu bestimmten sozialen Gruppen oder Institutionen [...] gleichsam eine Rendite $a b \ll$ (Peter, 2004, S. 52f.). Die Person erhält beispielsweise leichteren Zugang zu Bildung, zu Forschung und den entsprechenden Institutionen.

Um symbolische Gewalt auszuüben, ist aber nach Bourdieu zuvor ein Akt der Anerkennung oder Abwertung notwendig, der sich aus dem symbolischen Kapital ergibt. 
In Fortführung des Beispiels der Exklusion heißt das: Die Person mit Behinderung erhält beispielsweise Anerkennung durch die Möglichkeit, eine Stelle an einer Universität anzutreten, muss sich dort aber erneut Achtung oder Akzeptanz verschaffen, indem sie sich Akten der symbolischen Gewalt unterwirft. Das kann konkret heißen, sich in bestimmte Hierarchien zu fügen oder Ausgrenzungsversuche vonseiten der Student_innen oder Kolleg_innen hinzunehmen (Kubanski, 2013, S. 49ff.)

Symbolische Gewalt wird von Lothar Peter (2004, S. 48) auch als »sanfte Gewalt « beschrieben, um zum Ausdruck zu bringen, dass es sich hier nicht um physische Gewalt oder offene Repressalien handelt. Auch in distanzlosen oder herabwürdigenden Blicken können sich z. B. Akte der symbolischen Gewalt zeigen, da sie einen Menschen still verhöhnen oder Schamgefühle hervorrufen können (Prütting, 2006, S. 124).

Macht und Praxen symbolischer Gewalt können bewirken, dass sich Subjekte, hier z. B. Menschen mit einer Behinderung, selbsttätig und wie von allein in gesellschaftliche Formationen einfügen. Macht und symbolische Gewalt sind daher bedeutsame Kategorien für die Analyse, um unsichtbare Strategien, Techniken und Versprechen sichtbar werden zu lassen (Bourdieu, 1998; Peter, 2004).

Handlungen symbolischer Gewalt ergeben sich demnach aus der habituellen Abstimmung der Akteure mit ihrem Gegenüber, das heißt, auch (behinderte) Frauen, Männer und Transmenschen haben möglicherweise Dispositionen verinnerlicht, wie etwa eine habituelle Scham oder ein Gefühl der Minderwertigkeit. Nicht behinderte Akteure haben zuvor dafür die Maßstäbe festgelegt, mit denen z. B. Leistung und Können gemessen wird. Sie bestimmen die Norm und geben damit Anlass zur Beschämung. Akte symbolischer Gewalt reproduzieren sich genau an dieser Stelle immer wieder neu und können die Beteiligung Betroffener oder den Einbezug von Expert_innen in eigener Sache im Forschungskontext erschweren. An dieser Stelle wirken verschiedene Aspekte von Ungleichheit und eine intersektionale Betrachtungsweise böte Erklärungsmöglichkeiten, denn gerade das Verwobensein von Differenzkategorien ist beachtenswert. Dieselben Differenzen können »je nach Kontext und je nach Deutungs- und Handlungsmacht der an diesen Kontexten beteiligten Personen oder Gruppen [...] trivial oder sinnvoll, bloß interessant oder existenziell bedeutsam sein « (Hark \& Villa, 2017, S. 12f.).

Symbolische Gewalt findet sich nach Bourdieu vor allem dort, wo die Anwendung offener Gewalt nicht möglich ist, weil bestimmte Gesetze oder Konventionen dies untersagen (Peter, 2004, S. 48f.). Es wird die These vertreten, dass Hochschulen und ihre Forschungskontexte Räume bilden, die den Einsatz symbolischer Gewalt begünstigen.

Ein einseitiges Machtverständnis, dass z. B. Frauen mit Behinderung in der Opferrolle sehen, gilt es zu überwinden, um ihr Handlungspotenzial offenzulegen. Sinnvoll erscheint die Konzeptualisierung von Macht früherer feministischer Theoretikerinnen, wie Amy Allen (1998) oder Patricia H. Collins (1993). Deren analytische Unterschei- 
dung zwischen »power-over «, » power-to « und »power-with « wird von Bargetz et al. (2017) aktuell wieder aufgegriffen. Diese »soll insbesondere kollektive Handlungsfähigkeit und feministische Solidarität greif- und denkbar machen« (ebd., S. 12). Intersektionale Ansätze »verabschieden sich daher von der Vorstellung, dass das Verhältnis von Macht und Geschlecht in einer >Entweder-Oder-Logik< gefasst werden kann (Macht haben oder nicht), und machen deutlich, dass das Verhältnis erst durch eine $>$ Sowohl-als-auch-Perspektive $<$ durchdrungen werden kann (Macht haben und nicht haben) (vgl. Collins, 1993, 28f.) « (zit.n. ebd., S. 16). Diesen Überkreuzungen gilt es in der partizipativen Forschung verstärkt Aufmerksamkeit zu schenken.

\section{Rahmenbedingungen und Möglichkeiten partizipativer Forschung}

Wenn partizipative Forschung realisiert werden soll, dann müssen - wie wir herausgestellt haben - alle am Forschungsprozess Beteiligten bereit sein, Macht abzugeben, um Offenheit, Verschiedenheit und Emanzipationsprozesse zuzulassen. Dafür sollten sich im Besonderen die institutionellen Hierarchiestrukturen der Hochschule öffnen, aber auch die der anderen beteiligten Organisationen. Im Forschungsprozess müssen Entscheidungen zwischen allen Beteiligten ausgehandelt werden. Nur wenn ein Konsens gefunden werden kann, besteht eine realistische Chance, den Prozess fortzusetzen. Es gilt subtile Mechanismen der Exklusion aufzuspüren und die jeweiligen Fremdinteressen aufzudecken. Die nachfolgenden Beispiele zeigen, welche gesellschaftspolitischen Interessen hier wirken können.

Carolin Kollewe (2015) verweist auf Studien im angelsächsischen Raum und macht darauf aufmerksam, dass

»partizipative Projekte dazu beitragen, soziale Ungleichheit zu reproduzieren: Es ist vor allem die gebildete Mittelschicht, die über das kulturelle Kapital verfügt, sich in solche Prozesse einzubringen, da anderen die zeitlichen und kulturellen Ressourcen (z. B. rudimentäre Sachkenntnis, rhetorische Fähigkeiten, Selbstbewusstsein) für solch ein Engagement oftmals fehlt« (Kollewe, 2015, o.S.).

Ökonomisches Kapital, soziales Kapital, physisches, juridisches und symbolisches Kapital sind gleichsam bedeutsam für Beteiligungsprozesse (Goeke, 2010). Kollewe (2015) sieht am Beispiel der partizipativen Forschung und der Beteiligungsformen alter Menschen in Großbritannien die Diskurse um Partizipation eng mit dem Umbau des Wohlfahrtsstaates zu einem aktivierenden Sozialstaat und neoliberalen Politikansätzen verknüpft. 
Wie wird folglich im Forschungsbetrieb verallgemeinerbares Wissen produziert und in welchem Ausmaß erlangt es Gültigkeit? Diese Fragen ernst zu nehmen bedeutet, die begrenzte Reichweite von Erkenntnissen wörtlich zu nehmen. Holtmann meint ohnehin, dass durch Forschung entstandenes Wissen ein »zeitlich/räumlich/quantitativ begrenztes und nicht universell gültiges ist « (Holtmann, 2015, S. 98).

Komplizierte Sprache kann ebenfalls als institutionelles Machtinstrument (unbewusst) genutzt werden und stellt eine Barriere dar, um symbolische Gewalt auszuüben. Was würde passieren, wenn Hochschulen und Universitäten ein Angebot in einfacher Sprache vorhalten würden, ihre Homepages durchweg barrierefrei gestalten müssten? Hochschulen mit Lehrstühlen für Disability Studies haben dies bereits umgesetzt, für Studiengänge mit Inklusionsprofessor_innen ist dies keineswegs Standard. Im Verständnis einer inklusionsorientierten Hochschule könnte darüber hinaus Inklusion als Bewusstseinshaltung etabliert und eine inklusive Forschungs- und Lehrkultur etabliert werden (Hauser \& Schuppener, 2015). Menschen mit Behinderung wären so nicht nur Studierende, sondern sie träten selbstverständlich auch als Forschende und Lehrende an der Hochschule auf. Lehrtätigkeiten würde konsequenterweise auch durch Lehrende mit Lernschwierigkeiten gestaltet, sodass sie Aufgaben übernehmen, die bislang Akademiker_innen vorbehalten waren (ebd.).

Ein Beispiel, das zugleich eine machtkritische Perspektive auf Schule einnimmt und intersektionalen Perspektiven zwischen Männlichkeit und Fähigkeit nachgeht, ist das Forschungsprojekt »Inclusive Spaces «. In dessen Rahmen wird das Zusammenspiel von Männlichkeit und Fähigkeit an der Neuen Mittelschule in Wien untersucht und »die Verhandlungen von Fähigkeit und Männlichkeit aus einer theoretischen Perspektive heraus betrachtet, die sich aus dem fähigkeitskritischen Konzepten der Dis/Ability Studies, dem Modell hegemonialer Männlichkeit (Connell, 2002, 1995) sowie raumsoziologischen Überlegungen generiert « (Buchner, 2017, S. 2). Neben dem Fokus auf die Verwobenheit von Fähigkeit, Männlichkeit und Raum ist der forschungsmethodische Zugang inspirierend für unsere Betrachtung. Denn in einem weiterführenden Schritt werden in diesem Forschungsprojekt Kinder und Jugendliche im Alter von zehn bis14 Jahren befähigt, eigene Forschungsprojekte zu Raum, Differenz und Schule durchzuführen (ebd., S. 7). Dazu setzen sie sich zuvor spielerisch mit den Grundlagen partizipativer Forschung auseinander. Durch die Erforschung sozialer Räume an ihrer Schule nehmen sie nicht nur die Expertenrolle ein, sondern gewinnen zugleich symbolisches Kapital.

\section{Resümeé: Den Weg frei machen ...}

Entlang unserer Ausführungen und Beispiele ist deutlich geworden, dass die Rahmenbedingungen für eine gelingende Partizipation an Forschungsprozessen (keine Alibi- 
Partizipation) zu Beginn formuliert und benannt werden müssen. Ziel ist es, das große Potenzial von sehr unterschiedlichen Menschen als Bürger_innenwissen zu nutzen, denn von Bürger_innen zu sprechen schließt alle Menschen einer Gesellschaft ein. $\mathrm{Da}$ in der Gesellschaft implizite und explizite Machtpraktiken permanent wirksam sind, sind diese abzugeben bzw. power sharing zu praktizieren ein zentrales Moment. Hierin liegt die Chance, Macht positiv zu konnotieren und in ihr ein Potenzial für Veränderungen zu sehen, vor allem als Möglichkeit für gemeinsames politisches Handeln. Art und Weise der Partizipation von Bürger_innen kann je nach Forschungsfrage und Kontext variieren. Partizipative Forschung möchte jedoch größtmögliche Gestaltungsmacht und Entscheidungsmacht in die Hände von Bürger_innen legen und erreichen, dass Wissenschaftler_innen und Bürger_innen gemeinsam (forschend) handeln.

Im Kontext unserer Überlegungen sollte partizipative Forschung konsequent intersektional angelegt werden, um der Heterogenität der Menschen gerechter zu werden und um subtile Ausschlüsse nachzuvollziehen. Vertreter_innen des Konzepts Intersektionalität möchten additive Perspektiven - wie z.B. doppelte Diskriminierung überwinden und den Fokus auf das dynamische Zusammenwirken von sozialen Ungleichheiten lenken. Die Abkehr von eindimensionalen, monokausalen Erklärungen von Ungleichheit und Differenz ist notwendig und mit dem Konzept der Intersektionalität liegt eine sinnvolle Konzeptualisierung vor (Jacob et al., 2010). Hierbei ist zentral zu reflektieren, welche Kategorien betont und welche eher ausgeklammert werden.

Erkenntnisleitend ist es, grundlegende Exklusionsmechanismen zu verstehen und gesellschaftliche Organisationsprinzipien von Produktion und Reproduktion zu erkunden. Partizipative Forschungszugänge leisten einen Beitrag dazu, unter der Voraussetzung einer selbstreflexiven Haltung als privilegierte Forscher_innen in dem Prozess der Wissensproduktion. Deutlich wird, dass das gemeinsame Forschungshandeln eine wertorientierte Haltung impliziert. Teilhabe, Anerkennung und Empowerment sind dabei zentrale Werte.

Unabdingbar ist zudem die kritische Reflexion von Sprecher_innenpositionen und Repräsentationen im partizipativen Forschungsprozess, und dies gilt ausdrücklich auch für uns als die Autorinnen dieses Beitrags: Wer spricht? Für wen? Zu welchem Zweck? Wer wird adressiert? Zu Beginn partizipativer Forschungsprozesse sollte immer die Klärung und Reflexion des zugewiesenen Expert_innenstatus stehen: Wer ist für was Experte oder Expertin? - Wir, die Autorinnen, sind Frauen und nicht behindert, haben aber Forschungserfahrungen mit Frauen mit Behinderung. Somit sind wir als Autorinnen dem Forschungsgegenstand und dem Thema des Beitrags in besonderer Weise verbunden und treten für die Umsetzung von respektvollem und nicht vereinnahmendem Schreiben ein. 
Für den Prozess selbst ist relevant: Welche Kompetenzen brauche ich für ein diversitätsbewusstes und diskriminierungskritisches Forschungshandeln? Wer wird durch welche Institutionen anerkannt, unterstützt und befäbigt? Welche Potenziale, aber auch Grenzen des gemeinsamen Forschens bestehen?

Wenn alle diese Fragen genügend Raum bekommen, benötigt ein partizipatives Forschungsprojekt mehr Zeit und die Bereitschaft zu einer offenen Fehlerkultur sowie die Offenheit, neue Wege in der akademischen Forschung zu gehen. Der Gefahr einer Einengung durch zunehmende Ökonomisierung müssen neue Vorgehensweisen entgegengesetzt werden.

Solange strukturelle und gesellschaftliche Benachteiligungen immer wieder aus dem Blick geraten und Behinderung als defizitär konnotiert und individuelle Beeinträchtigung dargestellt wird, ist es noch ein Stück Weg hin zu echter partizipativer Forschung. Interdependenz zu denken bietet die Chance, alle Menschen mit Unterschieden und Ungleichheiten wahrzunehmen, ungewöhnliche Räume zu betreten und tradierte Rollen aufzulösen. Machen wir den Weg frei für neue Denkweisen in der Forschungslandschaft.

\section{Anmerkung}

1 Bourdieu beschreibt zwei Seiten im Habitus eines Menschen. Die strukturierende Struktur ist als eine Seite des Habitus für Bourdieu die Möglichkeit zur Veränderung, während die strukturierte Struktur die sozialisatorische Geschichte eines Menschen ist und damit auch weniger veränderbar zu sein scheint (Bourdieu, 1993, S. 113).

\section{Literatur}

Aktionsbündnis Teilhabeforschung (2015). Informationenzuallen Veranstaltungen,Zugang zu Arbeitspapieren und Redebeiträgen. https://teilhabeforschung.bifos.org/index.php/veranstaltungen (18.02.2018).

Allen, A. (1998). Rethinking power. Hypatia, 13(1), 21-40.

Arbeitsgemeinschaft Disability Studies Deutschland (2018). http://www.disabilitystudies.de/(16.02.2018). Bargetz, B., Lepperhoff, J., Ludwig, G., Scheele, A. \& Wilde, G. (2017). Geschlechterverhältnisse als Machtverhältnisse. FEMINA POLITICA - Zeitschrift für Feministische Politikwissenschaften, 26(1), 11-24.

Bergold, J.\& Thomas, S. (2012). Partizipative Forschungsmethoden: Ein methodischer Ansatz in Bewegung. Forum Qualitative Sozialforschung/Forum: Qualitative Social Research, 13(1), Art. 30. http:// nbn-resolving.de/urn:nbn:de:0114-fqs1201302 (24.09.2017).

Bourdieu, P. (1993). Sozialer Sinn - Kritik der theoretischen Vernunft. Frankfurt am Main: Suhrkamp. Bourdieu, P. (1998). Praktische Vernunft. Frankfurt am Main: Suhrkamp.

Buchner, T. (2017). »Ma' merkt auch, dass Maksim in der Klasse die Macht hat«: Zur sinkludierenden Wirkung hegemonialer Männlichkeit in den nicht inklusiven Räumen von Schule. Zeitschrift FürInklusion, 1(4). https://www.inklusion-online.net/index.php/inklusion-online/article/view/451 (24.03.2018). 
Dagmar Kubanski \& Stephanie Goeke: Das Verhältnis von Macht, Geschlecht und (Dis-)Ability ...

Buchner, T. \& Koenig, O. (2013). Von einem, der Wege geebnet hat ... In P. Flieger \& S. Plangger (Hrsg.). (2013). Aus der Nähe. Zum wissenschaftlichen und behinderten politischen Wirken von Volker Schönwiese (S. 175-182). Neu-Ulm: AG Spak.

Buchner, T., Koenig, O. \& Schuppener, S. (Hrsg.). (2016). Inklusive Forschung. Gemeinsam Forschen mit Menschen mit Lernschwierigkeiten. Bad Heilbrunn: Julius Klinkhardt.

Collins, P. (1993). Toward a new vision: Race, class, and gender as categories of a analysis and connection. Race, Sex \& Class, 1(1), 25-45.

Cook, T., Atkin, H. \& Wilcockson, J. (2018). Particiaptory research into inclusive practise: Improving services for people with long time term neurological conditions. Forum Qualitative Sozialforschung/Forum: Qualitative Social Research, 19(1), Art. 4. http://dx.doi.org/10.17169/fqs-19.1.2667 (04.03.2018).

Deutsche Gesellschaft für Erziehungswissenschaft (DGfE) (2016). http://www.dgfe.de/sektionen -kommissionen/arbeitsgemeinschaft-inklusionsforschung.html (02.02.2018).

Evangelische Hochschule Rheinland-Westfalen-Lippe (2015). Disability Studies Deutschland. https:// bodys.evh-bochum.de/disability-studies.html (16.02.2018).

Gerhards, J. (2013). Der deutsche Sonderweg in der Messung von Forschungsleistungen. Berlin: BerlinBrandenburgische Akademie der Wissenschaften (Wissenschaftspolitik im Dialog, 7). http://nbn -resolving.de/urn/resolver.pl?urn:nbn:de:kobv:b4-opus-23876 (08.09.2018).

Goeke, S. (2010). Frauen stärken sich. Empowermentprozesse von Frauen mit Behinderungserfahrung. Marburg: Lebenshilfe-Verlag.

Goeke, S. \& Kubanski, D. (2012). Menschen mit Behinderungen als Grenzgängerlnnen im akademischen Raum - Chancen partizipatorischer Forschung. Forum Qualitative Sozialforschung/Forum: Qualitative Social Research, 13(1), Art. 6. http://nbn-resolving.de/urn:nbn:de:0114-fqs120162 (17.02.2018).

Grefe, C \& Sentker, A. (2014). Streit ums Mitspracherecht. Zeit Online. http://www.zeit.de/2014/39/ foerdermittel-forschungsprojekte-mitspracherecht (18.02.2018).

Hark, S. \& Villa, P.-I. (2017). Unterscheiden und Herrschen. Ein Essay zu den ambivalenten Verflechtungen von Rassismus, Sexismus und Feminismus in der Gegenwart. Bielefeld: transcript.

Hauser, M. \& Schuppener, S. (2015). Menschen mit Lernschwierigkeiten an der Hochschule. Entwicklungen in Großbritannien, Irland und Deutschland. Teilhabe, 54(3), 100-106.

Hinz, A. \& Köpfer A. (2016). Unterstützung durch Dekategorisierung? Beispiele für Unterstützung durch Dekategorisierung. Vierteljahresschrift für Heilpädagogik und Nachbargebiete (VHN), 1/2016, 36-47.

Hirschberg, M. (2015). Die überaus fähige Lehrkraft. Zur Wirkungsweise von Ableism in der Subjektivierung von Lehrkräften. Zeitschrift für Inklusion, (2). https://www.inklusion-online.net/index.php/ inklusion-online/article/view/274 (16.02.2018).

Holtmann, I. (2015). Verbündet-Sein: Grenzen und Möglichkeiten partizipativer Forschung. Wie Inter*Diskriminierungen verantwortungsvoll interdepenDenken aus privilegierter Position? In AK ForschungsHandeln (Hrsg.), InterdepenDenken! (S. 96-111). Berlin: W_orten \& meer GmbH.

Jacob, J., Köbsell, S. \& Wollrad, E. (Hrsg.). (2010). Gendering Disability. Intersektionale Aspekte von Behinderung und Geschlecht. Bielefeld: transcript.

Kollewe, C. (2015). »Nothing about us«? - Partizipative Forschung und Beteiligungsformen alter Menschen am Beispiel Großbritannien. Journal für Psycholologie, 23(1). https://www.journal-fuer -psychologie.de/index.php/jfp/article/view/343/400 (05.01.2018).

Köbsell, S. (2010). Gendering Disability: Behinderung, Geschlecht und Körper. In J. Jacob, S. Köbsell \& E. Wollrad (Hrsg.), Gendering Disability. Intersektionale Aspekte von Behinderung und Geschlecht (S. 17-33). Bielefeld: transcript.

Kubanski, D. (2013). Grenzüberschreitungen im Leben von Frauen mit Behinderung. Eine qualitative Studie zur Konstruktion von Selbstbehauptungsstrategien im Alltag behinderter Frauen im Kontext grenz- 
überschreitender Situationen. Freiburg, Pädagogische Hochschule, Diss. https://phfr.bsz-bw.de/ frontdoor/index/index/docld/396 (06.01.2018).

Ludwig, G. (2011). Kritik als Haltung unter universitären Bedingungen. Kurswechsel, (1), 117-125. http:// www.beigewum.at/wordpress/wp-content/uploads/Kritik-als-Haltung-universit\%C3\%A4rer -Bedingungen.pdf (01.07.2018).

Maskos, R. (2010). Was heißt Ableism? Überlegungen zu Behinderung und bürgerlicher Gesellschaft. arranca!, 43. http://arranca.org/43/was-heisst-ableism (28.01.2018).

Maskos, R. (2015). Ableism und das Ideal des autonomen Fähig-Seins in der kapitalistischen Gesellschaft. Zeitschrift für Inklusion, (2). https://www.inklusion-online.net/index.php/inklusion-online/ article/view/277 (22.03.2018).

Mehrholz, J. (2018). Frühzeitige Versorgung. https://www.ottobock.de/media/lokale-medien-de_de/ neurorehabilitation/files/neurorehabilitation_special_2018.pdf (08.09.2018).

Peter, L. (2004). Pierre Bourdieus Theorie der symbolischen Gewalt. In M. Steinrücke (Hrsg.), Pierre Bourdieu. Politisches Forschen, Denken und Eingreifen (S. 48-73). Hamburg: VSA-Verlag.

Pfahl, L. (2014). »Ableism. Behinderung und Befähigung auf der Spur«. Vortrag auf der Tagung »Young Disability Pride am 8. November 2014. http://www.isl-ev.de/attachments/article/1166/ B-\%20Vortrag\%20Lisa\%20Pfahl_Ableism-Behinderung\%20und\%20Befähigung\%20auf\%20der\% 20Spur.pdf (05.11.2017).

Prütting, Lenz (2006). »Und auf Vernichtung läuft's hinaus.« Über Gelächter und Scham. Berliner Debatte Initial, 17, (1-2): Mitgefühl/Scham und Macht, 123-136.

Raab, H. (2010). Shifting the paradigm: »Behinderung, Heteronormativität und Queerness«. In J. Jacob, S. Köbsell \& E. Wollrad (Hrsg.), Gendering Disability. Intersektionale Aspekte von Behinderung und Geschlecht (S. 73-94). Bielefeld: transcript.

Raab, H. (2015). Intersektionalität revisited. Vortrag am 26.01.2015 im Rahmen der Ringvorlesung »Behinderte ohne Behinderte?! Perspektiven der Disability Studies«. http://www.zedis-ev-hochschule -hh.de/files/raab_260115.pdf (22.03.2018).

Reichenbach, C. (2009). Diagnostik und Förderung des Selbstkonzeptes als pädagogische Aufgabe. Integrationsjourna Wienl, 76-85.

Russo, J. (2012). Vorwort. In Verein zum Schutz vor psychiatrischer Gewalt e.V. (Hrsg.), Auf der Suche nach dem Rosengarten. Projektdokumentation (S. 2-3). Berlin: Agentur TEKTEK.

Ministerium für Kultur und Wissenschaft des Landes Nordrhein-Westfalen (2018). Inter- und transdisziplinäre Forschung. https://www.mkw.nrw/forschung/fortschritt-nrw/inter-und-transdisziplinaere -forschung/ (04.03.2018).

UN-Behindertenrechtskonvention. https://www.behindertenrechtskonvention.info/ (04.03.2018).

Unger, H. von (2014). Partizipative Forschung. Einführung in die Forschungspraxis. Wiesbaden: Springer VS.

Waldschmidt, A. (2005). Disability Studies: Individuelles, soziales und/oder kulturelles Modell von Behinderung? Psychologie \& Gesellschaftskritik, 29, 9-31.

Waldschmidt, A. (2015). Grundlagen und Ziele der Teilhabeforschung. Lebenslage und Partizipation von Menschen mit Behinderungen. Sozialrecht + Praxis. Fachzeitschrift für Sozialpolitiker und Schwerbehindertenvertreter, 25(11), 683-688.

Walmsley, J. (2001). Normalisation, Emancipatory Research and Inclusive Research in Learning Disability. Disability \& Society, 16(1), 187-205.

Walmsley, J. \& Johnson, K. (2003). Inclusive research with people with learning disabilities. Past, present and futures. London, New York: Jessica Kingsley.

Zander, M. (2016). Disability Studies: Gesellschaftliche Ausgrenzung als Forschungsgegenstand. Bundesgesundheitsblatt, 59(9), 1048-1052.

Zarb, G. (1992). On the road to damascus: first steps towards changing the relations of disability research production. Disability, Handicap \& Society, 7(2), 125-138. 


\section{Die Autorinnen}

Dagmar Kubanski, Dr. phil., ist als wissenschaftliche Mitarbeiterin im Studiengang Bachelor of Nursing an der Evangelischen Hochschule Berlin tätig. Sie ist Mitglied der Arbeitsgruppe Partizipative Teilhabeforschung \& Forschungsmethoden im Aktionsbündnis Teilhabeforschung, und Mitglied des Präsidiums des Berliner Behindertenund Rehabilitationssportverbandes e. V. (Bereich: Bildung).

Kontakt:kubanski@eh-berlin.de

Stephanie Goeke, Dr. phil., arbeitet als Referentin im Caritasverband der Diözese Rottenburg-Stuttgart e. V. und ist nebenberuflich in Praxisentwicklungsforschung, Hochschullehre und Projektberatung tätig.

Kontakt: stephaniegoeke@gmx.de 